OPEN ACCESS

Edited by:

Yibin Feng,

The University of Hong Kong,

Hong Kong

Reviewed by:

Qibiao Wu

Macau University of Science and Technology, Macau

Di Wang,

Jilin University, China

${ }^{*}$ Correspondence:

Rui Zhao

3201039@zju.edu.cn

Specialty section:

This article was submitted to Pharmacology of Anti-Cancer Drugs,

a section of the journa

Frontiers in Oncology

Received: 26 May 2020 Accepted: 17 September 2020

Published: 28 October 2020

Citation:

Li M, Ying M and Zhao R (2020) Commentary: Efficacy and Safety of Chinese Herbal Medicine on Ovarian Cancer After Reduction Surgery and Adjuvant Chemotherapy: A Systematic

Review and Meta-Analysis.

Front. Oncol. 10:565812.

doi: 10.3389/fonc. 2020.565812

\section{Commentary: Efficacy and Safety of Chinese Herbal Medicine on Ovarian Cancer After Reduction Surgery and Adjuvant Chemotherapy: A Systematic Review and Meta-Analysis}

\author{
Mingxing Li, Miaofa Ying and Rui Zhao* \\ Department of Pharmacy, Sir Run Run Shaw Hospital, School of Medicine, Zhejiang University, Hangzhou, China
}

Keywords: Chinese herbal medicine, ovarian cancer, meta-analysis, comment analysis, efficacy and safety

\section{A Commentary on}

Efficacy and Safety of Chinese Herbal Medicine on Ovarian Cancer After Reduction Surgery and Adjuvant Chemotherapy: A Systematic Review and Meta-Analysis

by Wang, R., Sun, Q., Wang, F., Liu, Y., Li, X., Chen, T., et al. (2019). Front. Oncol. 9:730. doi: $10.3389 /$ fonc.2019.00730

In a previous issue of Frontiers in Oncology published in August 2019, we read with great interest the article by Wang et al. (1) entitled "Efficacy and Safety of Chinese Herbal Medicine on Ovarian Cancer After Reduction Surgery and Adjuvant Chemotherapy: A Systematic Review and Meta-Analysis." The authors performed a meta-analysis to assess the efficacy and safety of Chinese herbal medicine (CHM) in the treatment of ovarian cancer after reduction surgery and adjuvant chemotherapy. The study is of great value and provides a proper regimen for the treatment of ovarian cancer in the future. However, there are still some flaws that we would like to discuss with the authors.

First, only two electronic databases (CNKI and PubMed) were searched by the authors, and 18 studies from China were included. According to the Preferred Reporting Items for Systematic Reviews and Meta-Analysis statement criteria (2), the proper search strategies and adequate studies are essential when reporting a meta-analysis. Therefore, we suggested that more electronic databases should be systematically searched, including the Wanfang database, Cochrane Library, Embase, and Medline, and detailed search protocols should be provided by the authors.

Second, all the included studies came from Chinese journals in this study, and the details of quality assessment of all the studies were not provided. In order to ensure the accuracy of the results, all studies should be scored according to the Jadad Scale (Table 1). In addition, according to the Cochrane collaboration's tool (3), the methodological quality and the risk of bias of each study should be evaluated by two investigators. However, the authors did not conduct the evaluation in their meta-analysis. In our opinion, the methodological quality and the risk of bias should be performed so as to eliminate the low-quality studies. 
TABLE 1 | Jadad scale.

\begin{tabular}{|c|c|c|c|}
\hline \multirow[t]{2}{*}{ Items } & \multicolumn{3}{|c|}{ Scores (0-7) } \\
\hline & 0 & 1 & 2 \\
\hline Randomization & $\begin{array}{l}\text { Not randomized or inappropriate method of } \\
\text { randomization }\end{array}$ & The study was described randomized & $\begin{array}{l}\text { The method of randomization was described, } \\
\text { and it was appropriate }\end{array}$ \\
\hline Double blinding & No blind or inappropriate method of blinding & The study was described double blind & $\begin{array}{l}\text { The method of double blinding was } \\
\text { described and it was appropriate }\end{array}$ \\
\hline Concealment of allocation & $\begin{array}{l}\text { Not describe the method of allocation } \\
\text { concealment }\end{array}$ & $\begin{array}{l}\text { The study was described allocation } \\
\text { concealment }\end{array}$ & $\begin{array}{l}\text { The method of allocation concealment was } \\
\text { described, and it was appropriate }\end{array}$ \\
\hline Withdrawals and dropouts & Not describe the follow-up & $\begin{array}{l}\text { The study was described withdrawals and } \\
\text { dropouts }\end{array}$ & \\
\hline
\end{tabular}

Third, Q test and $I^{2}$ test were used to assess heterogeneity in their meta-analysis. According to the Cochrane Reviewers' Handbook, the fixed effect model was used when $I^{2}$ value was less than $50 \%$, otherwise the random effect model would be applied. In their article, subgroup analyses were used to evaluate the urinary system symptoms, and the results are shown in Figure 3C. Although the values of $I^{2}$ were no more than $50 \%$, the random effect models were still used by the authors in Figures 3C,D,F. We hope to get the authors' viewpoint on this issue. In addition, the results of peripheral neuropathy are showed in Figure 3E, and not in Figure 3F, a mislabeling. Meanwhile, we think that sensitivity analysis is still necessary to ensure the accuracy of the results.

Fourth, even though the risk of bias was assessed using the Cochrane risk of bias tool (4), the publication bias

\section{REFERENCES}

1. Wang R, Sun Q, Wang F, Liu Y, Li X, Chen T, et al. Efficacy and safety of Chinese herbal medicine on ovarian cancer after reduction surgery and adjuvant chemotherapy: a systematic review and meta-analysis. Front Oncol. (2019) 9: 730. doi: 10.3389/fonc.2019.00730

2. Moher D, Liberati A, Tetzlaff J, Altman DG, PRISMA Group. Preferred reporting items for systematic reviews and meta-analyses: the PRISMA statement. PLoS Med. (2009) 7:e1000097. doi: 10.1371/journal.pmed.10 00097

3. Higgins JPT, Altman DG, Gotzsche PC, Jüni P, Moher D, Oxman AD, et al. The Cochrane Collaboration's tool for assessing risk of bias in randomised trials. BMJ. (2011) 343:d5928. doi: 10.1136/bmj.d5928 was not mentioned in their meta-analysis. As far as we know, the funnel plots should be used to evaluate the publication bias.

All in all, we are grateful to the authors for their contribution; they summarized the safety and efficacy of $\mathrm{CHM}$ in the treatment of ovarian cancer after reduction surgery and adjuvant chemotherapy. However, rigorous preclinical high-quality RCTs involving CHM in the treatment of ovarian cancer is still needed to reach rational conclusions.

\section{AUTHOR CONTRIBUTIONS}

All authors listed have made a substantial, direct and intellectual contribution to the work, and approved it for publication.

4. Dersimonian R, Laird N. Meta-analysis in clinical trials. Control Clin Trials. (1986) 7:177-88.

Conflict of Interest: The authors declare that the research was conducted in the absence of any commercial or financial relationships that could be construed as a potential conflict of interest.

Copyright (c) $2020 \mathrm{Li}$, Ying and Zhao. This is an open-access article distributed under the terms of the Creative Commons Attribution License (CC BY). The use, distribution or reproduction in other forums is permitted, provided the original author(s) and the copyright owner(s) are credited and that the original publication in this journal is cited, in accordance with accepted academic practice. No use, distribution or reproduction is permitted which does not comply with these terms. 